Proyecciones Journal of Mathematics

Vol. 29, No 2, pp. 123-135, August 2010.

Universidad Católica del Norte

Antofagasta - Chile

\title{
COUNTABLE COMPACTNESS AND THE \\ LINDELOF PROPERTY IN L-FUZZY TOPOLOGICAL SPACES *
}

\author{
$R U N-X I A N G L I$ \\ BEIJING INSTITUTE OF TECHNOLOGY, CHINA \\ and \\ $F U$ - GUI SHI \\ BEIJING INSTITUTE OF TECHNOLOGY, CHINA \\ Received : April 2010. Accepted : May 2010
}

\begin{abstract}
In this paper, the concepts of L-fuzzy countable compactness and the L-fuzzy Lindelöf property are introduced in L-fuzzy topological spaces, where $L$ is a completely distributive DeMorgan algebra. An $L$-fuzzy compact L-fuzzy set is L-fuzzy countably compact and has the L-fuzzy Lindelöf property. An L-fuzzy set having the L-fuzzy Lindelöf property is L-fuzzy countably compact if and only if it is L-fuzzy compact. Many characterizations of L-fuzzy countable compactness and the L-fuzzy Lindelöf property are presented.
\end{abstract}

Keywords : L-fuzzy topology, L-fuzzy countable compactness, the L-fuzzy Lindelöf property.

2000 Mathematics Subject Classification : 05C50, 15A03, $52 B 40$

\footnotetext{
${ }^{*}$ The project is supported by the National Natural Science Foundation of China (10971242).
} 


\section{Introduction}

In 1976, the concept of fuzzy compactness was introduced in [0,1]-topological spaces by R. Lowen [5]. Subsequently its characterization was given by G.J. Wang in terms of $\alpha$-net in [12]. In 1988, it was extended to $L$-topological spaces [13], where $L$ is a completely distributive DeMorgan algebra. In [9], a new definition of fuzzy compactness was presented by means of open $L$-sets and their inequality in $L$-topological spaces. When $L$ is a completely distributive DeMorgan algebra, it is equivalent to the notion of fuzzy compactness in $[4,7,13]$. Recently the concept of $L$-fuzzy compactness was introduced by Shi and Li [10] in $L$-fuzzy topological spaces.

In this paper, our aim is to continue the research of $L$-fuzzy countable compactness and the $L$-fuzzy Lindelöf property of $L$-fuzzy sets.

\section{Preliminaries}

Throughout this paper $\left(L, \bigvee, \bigwedge,^{\prime}\right)$ is a completely distributive DeMorgan algebra, $X$ is a nonempty set. $L^{X}$ is the set of all $L$-fuzzy sets on $X$. The smallest element and the largest element in $L^{X}$ are denoted respectively by $\perp$ and $I$. An $L$-fuzzy set is briefly written as an $L$-set. We often do not distinguish a crisp subset $A$ from its characteristic function $\chi_{A}$.

The set of nonunit prime elements in $L$ is denoted by $P(L)$. The set of nonzero co-prime elements in $L$ is denoted by $M(L)$. The set of nonzero co-prime elements in $L^{X}$ is denoted by $M\left(L^{X}\right)$. The set all $L$-fuzzy points $x_{\lambda}$ (i.e., an $L$-fuzzy set $A \in L^{X}$ such that $A(x)=\lambda \neq 0$ and $A(y)=0$ for $y \neq x)$ is denoted by $p t\left(L^{X}\right)$.

The binary relation $\prec$ in $L$ is defined as follows: for $a, b \in L, a \prec b$ if and only if for every subset $D \subseteq L, b \leq \sup D$ always implies the existence of $d \in D$ with $a \leq d$ [1]. In a completely distributive DeMorgan algebra $L$, each member $b$ is a sup of $\{a \in L \mid a \prec b\}$. In the sense of $[4,13]$, $\{a \in L \mid a \prec b\}$ is the greatest minimal family of $b$, denoted by $\beta(b)$, and $\beta^{*}(b)=\beta(b) \cap M(L)$. Moreover for $b \in L$, define $\alpha(b)=\left\{a \in L \mid a^{\prime} \prec b^{\prime}\right\}$ and $\alpha^{*}(b)=\alpha(b) \cap P(L)$.

For $a \in L$ and $A \in L^{X}$, we define $A_{[a]}=\{x \in X \mid A(x) \geq a\}$.

Definition $2.1([\mathbf{2}, \mathbf{3}, \mathbf{6}, \mathbf{1 1}])$. An $L$-fuzzy topology on a set $X$ is a map $\mathcal{T}: L^{X} \rightarrow L$ such that

(1) $\mathcal{T}(\underline{\top})=\mathcal{T}(\underline{\perp})=\top$;

(2) $\forall U, V \in L^{X}, \mathcal{T}(U \wedge V) \geq \mathcal{T}(U) \wedge \mathcal{T}(V)$; 
(3) $\forall U_{j} \in L^{X}, j \in J, \mathcal{T}\left(\bigvee_{j \in J} U_{j}\right) \geq \bigwedge_{j \in J} \mathcal{T}\left(U_{j}\right)$.

$\mathcal{T}(U)$ can be interpreted as the degree to which $U$ is an open set. $\mathcal{T}^{*}(U)=$ $\mathcal{T}\left(U^{\prime}\right)$ will be called the degree of closedness of $U$. The pair $(X, \mathcal{T})$ is called an $L$-fuzzy topological space.

A mapping $f:(X, \mathcal{T}) \rightarrow(Y, \mathcal{U})$ is said to be $L$-fuzzy continuous if $\mathcal{T}\left(f_{L}^{\leftarrow}(B)\right) \geq \mathcal{U}(B)$ holds for all $B \in L^{Y}$, where $f_{L}^{\leftarrow}$ is defined by $f_{L}^{\leftarrow}(B)(x)=B(f(x))[6]$.

Theorem $2.2([\mathbf{1 4}])$. Let $(X, \mathcal{T})$ and $(Y, \mathcal{U})$ be $L$-fuzzy topological spaces. Then $f:(X, \mathcal{T}) \rightarrow(Y, \mathcal{U})$ be $L$-fuzzy continuous if and only if $\forall a \in M(L)$, $f:\left(X, \mathcal{T}_{[a]}\right) \rightarrow\left(Y, \mathcal{U}_{[a]}\right)$ be L-continuous.

Definition 2.3 ([8, 9]). Let $a \in L \backslash\{\top\}$ and $G \in L^{X}$. A subfamily $U$ in $L^{X}$ is said to be

(1) an a-shading of $G$ if for any $x \in X$, it follows that $G^{\prime}(x) \vee \bigvee_{A \in \mathcal{U}} A(x) \not \leq$ $a$.

(2) a strong a-shading of $G$ if $\bigwedge_{x \in X}\left(G^{\prime}(x) \vee \bigvee_{A \in \mathcal{U}} A(x)\right) \not \leq a$.

Definition 2.4 ([8, 9]). Let $a \in L \backslash\{\perp\}$ and $G \in L^{X}$. A subfamily $\mathcal{P}$ in $L^{X}$ is said to be

(1) an a-remote family of $G$ if for any $x \in X$, it follows that $G(x) \wedge$ $\bigwedge_{B \in \mathcal{P}} B(x) \nsupseteq a$.

(2) a strong a-remote family of $G$ if $\bigvee_{x \in X}\left(G(x) \wedge \bigwedge_{B \in P} B(x)\right) \nsupseteq a$.

Definition 2.5 ([8, 9]). Let $a \in L \backslash\{\perp\}$ and $G \in L^{X}$. A subfamily $\mathcal{U}$ in $L^{X}$ is called

(1) a $\beta_{a}$-cover of $G$ if for any $x \in X$, it follows that $a \in \beta\left(G^{\prime}(x) \vee \bigvee_{A \in \mathcal{U}} A(x)\right)$

(2) a strong $\beta_{a}$-cover of $G$ if for any $x \in X$, it follows that

$a \in \beta\left(\bigwedge_{x \in X}\left(G^{\prime}(x) \vee \bigvee_{A \in \mathcal{U}} A(x)\right)\right)$. 
Definition 2.6 ([8, 9]). Let $a \in L \backslash\{\perp\}$ and $G \in L^{X}$. A subfamily $\mathcal{U}$ in $L^{X}$ is called a $Q_{a}$-cover of $G$ if $a \leq \bigwedge_{x \in X}\left(G^{\prime}(x) \vee \bigvee_{A \in \mathcal{U}} A(x)\right)$.

For a subfamily $\Phi \subseteq L^{X}, 2^{(\Phi)}$ denotes the set of all finite subfamilies of $\Phi .2^{[\Phi]}$ denotes the set of countable subfamilies of $\Phi$.

Definition $2.7([8])$. Let $(X, \mathcal{T})$ be an $L$-topological space. $G \in L^{X}$ is said to be countably compact if for every countable family $\mathcal{U} \subseteq L^{X}$, it follows that

$$
\bigwedge_{x \in X}\left(G^{\prime}(x) \vee \bigvee_{F \in \mathcal{U}} F(x)\right) \leq \bigvee_{V \in 2^{(\mathcal{U})}} \bigwedge_{x \in X}\left(G^{\prime}(x) \vee \bigvee_{F \in \mathcal{V}} F(x)\right)
$$

Definition $2.8([8])$. Let $(X, \mathcal{T})$ be an $L$-topological space. $G \in L^{X}$ is said to have the Lindelöf property if for every family $\mathcal{U} \subseteq L^{X}$, it follows that

$$
\bigwedge_{x \in X}\left(G^{\prime}(x) \vee \bigvee_{F \in \mathcal{U}} F(x)\right) \leq \bigvee_{\left.V \in 2^{[\mathcal{U}}\right]} \bigwedge_{x \in X}\left(G^{\prime}(x) \vee \bigvee_{F \in \mathcal{V}} F(x)\right) .
$$

Definition 2.9 ([10]). Let $(X, \mathcal{T})$ be an $L$-fuzzy topological space. $G \in$ $L^{X}$ is said to be $L$-fuzzy compact if for every family $\mathcal{U} \subseteq L^{X}$, it follows that

$$
\bigwedge_{F \in \mathcal{U}} \mathcal{T}(F) \wedge\left(\bigwedge_{x \in X}\left(G^{\prime}(x) \vee \bigvee_{F \in \mathcal{U}} F(x)\right)\right) \leq \bigvee_{\mathcal{V} \in 2^{(\mathcal{U})}} \bigwedge_{x \in X}\left(G^{\prime}(x) \vee \bigvee_{F \in \mathcal{V}} F(x)\right) .
$$

\section{3. $L$-fuzzy countable compactness}

Definition 3.1. Let $(X, \mathcal{T})$ be an $L$-fuzzy topological space. $G \in L^{X}$ is said to be $L$-fuzzy countably compact if for every countable family $\mathcal{U} \subseteq L^{X}$, it follows that

$$
\bigwedge_{F \in \mathcal{U}} \mathcal{T}(F) \wedge\left(\bigwedge_{x \in X}\left(G^{\prime}(x) \vee \bigvee_{F \in \mathcal{U}} F(x)\right)\right) \leq \bigvee_{\mathcal{V} \in 2^{(\mathcal{U})}} \bigwedge_{x \in X}\left(G^{\prime}(x) \vee \bigvee_{F \in \mathcal{V}} F(x)\right) .
$$

Obviously $L$-fuzzy compactness implies $L$-fuzzy countable compactness. Let $(X, \mathcal{T})$ be an $L$-topological space. Let $\chi_{\mathcal{T}}: L^{X} \rightarrow L$

$$
\chi_{\mathcal{T}}= \begin{cases}1, & A \in \mathcal{T} \\ 0, & A \notin \mathcal{T}\end{cases}
$$


Obviously, $\left(X, \chi_{\mathcal{T}}\right)$ is a special $L$-fuzzy topological spaces. So we can easily prove the following theorem.

Theorem 3.2. Let $(X, \mathcal{T})$ be an $L$-topological space and $G \in L^{X}$. $G$ is $L$-fuzzy countably compact in $\left(X, \chi_{\mathcal{T}}\right)$ if and only if $G$ is countably compact $[8]$ in $(X, \mathcal{T})$.

From Definition 2.1 we easily obtain the following theorem by simply using quasi-complement.

Theorem 3.3. Let $(X, \mathcal{T})$ be an $L$-fuzzy topological space. $G \in L^{X}$ is $L$ fuzzy countably compact if and only if for every countably family $\mathcal{P} \subseteq L^{X}$ it follows that

$$
\bigvee_{F \in \mathcal{P}} \mathcal{T}^{\prime}\left(F^{\prime}\right) \vee\left(\bigvee_{x \in X}\left(G(x) \wedge \bigwedge_{F \in P} F(x)\right)\right) \geq \bigwedge_{\mathcal{H} \in 2^{(P)}} \bigvee_{x \in X}\left(G(x) \wedge \bigwedge_{F \in H} F(x)\right) .
$$

By Definition 2.1 and Theorem 2.2 and analogous to [8] we immediately obtain the following result.

Theorem 3.4. Let $(X, \mathcal{T})$ be an $L$-fuzzy topological space and $G \in L^{X}$. Then the following conditions are equivalent to each other.

(1) $G$ is L-fuzzy countably compact.

(2) For any $a \in M(L)$, each countable strong a-remote family $\mathcal{P}$ of $G$ with $\bigwedge_{F \in P} \mathcal{T}^{*}(F) \not{Z} a^{\prime}$ has a finite subfamily $\mathcal{H}$ which is a (strong) a-remote family of $G$.

(3) For any $a \in M(L)$, and any countable strong a-remote family $\mathcal{P}$ of $G$ with $\bigwedge_{F \in P} \mathcal{T}^{*}(F) \not \leq a^{\prime}$, there exists a finite subfamily $\mathcal{H}$ of $\mathcal{P}$ and $b \in \beta^{*}(a)$ such that $\mathcal{H}$ is a (strong) b-remote family of $G$.

(4) For any $a \in P(L)$, each countable strong a-shading $\mathcal{U}$ of $G$ with $\bigwedge_{F \in \mathcal{U}} \mathcal{T}(F) \not \leq a$ has a finite subfamily $\mathcal{V}$ which is a (strong) a-shading of $G$.

(5) For any $a \in P(L)$ and any countable strong $a$-shading $\mathcal{U}$ of $G$ with $\bigwedge_{F \in U} \mathcal{T}(F) \not \leq a$, there exists a finite subfamily $\mathcal{V}$ of $\mathcal{U}$ and $b \in \alpha^{*}(a)$ such that $\mathcal{V}$ is a (strong) $b$-shading of $G$. 
(6) For any $a \in M(L)$ and any $b \in \beta^{*}(a)$, each countable $Q_{a}$-cover $\mathcal{U}$ of $G$ with $\mathcal{T}(F) \geq a(\forall F \in \mathcal{U})$ has a finite subfamily $\mathcal{V}$ which is a $Q_{b}$-cover of $G$.

(7) For any $a \in M(L)$ and any $b \in \beta^{*}(a)$, each countable $Q_{a}$-cover $\mathcal{U}$ of $G$ with $\mathcal{T}(F) \geq a(\forall F \in \mathcal{U})$ has a finite subfamily $\mathcal{V}$ which is a (strong) $\beta_{b}$-cover of $G$.

Theorem 3.5. Let $(X, \mathcal{T})$ be an $L$-fuzzy topological space and $G \in L^{X}$. If $\beta(c \wedge d)=\beta(c) \cap \beta(d)(\forall c, d \in L)$, then the following conditions are equivalent to each other.

(1) $G$ is $L$-fuzzy countably compact.

(2) For any $a \in M(L)$, each countable strong $\beta_{a}$-cover $\mathcal{U}$ of $G$ with $a \in$ $\beta\left(\bigwedge_{F \in \mathcal{U}} \mathcal{T}(F)\right)$ has a finite subfamily $\mathcal{V}$ which is a (strong) $\beta_{a}$-cover of $G$.

(3) For any $a \in M(L)$ and any countable strong $\beta_{a}$-cover $\mathcal{U}$ of $G$ with $a \in$ $\beta\left(\bigwedge_{F \in \mathcal{U}} \mathcal{T}(F)\right)$, there exists a finite subfamily $\mathcal{V}$ of $\mathcal{U}$ and $b \in M(L)$ with $a \in \beta^{*}(b)$ such that $\mathcal{V}$ is a (strong) $\beta_{b}$-cover of $G$.

Now in order to research properties of $L$-fuzzy countably compactness, we introduce the following definition.

Definition 3.6. Let $(X, \mathcal{T})$ be an $L$-topological space, $a \in M(L)$ and $G \in$ $L^{X} . G$ is said to be countably a-compact if and only if $\forall b \in \beta(a)$, each countable $Q_{a}$-open cover $\mathcal{U}$ of $G$ has a finite subfamily $\mathcal{V}$ which is a $Q_{b}$-open cover of $G$.

Theorem 3.7. Let $(X, \mathcal{T})$ be an $L$-topological space. $G \in L^{X}$ is countably compact if and only if $\forall a \in M(L), G$ is countably a-compact.

Theorem 3.8. Let $(X, \mathcal{T})$ be an $L$-fuzzy topological space and $G \in L^{X}$. $G$ is $L$-fuzzy countably compact in $(X, \mathcal{T})$ if and only if $\forall a \in M(L), G$ is countably a-compact in $\left(X, \mathcal{T}_{[a]}\right)$. 
Proof. (Necessity) Since $G$ is $L$-fuzzy countably compact in $(X, \mathcal{T})$, by Definition 2.1 we know that for every countable family $\mathcal{U} \subseteq L^{X}$, it follows that

$$
\bigwedge_{F \in \mathcal{U}} \mathcal{T}(F) \wedge\left(\bigwedge_{x \in X}\left(G^{\prime}(x) \vee \bigvee_{F \in \mathcal{U}} F(x)\right)\right) \leq \bigvee_{\mathcal{V} \in 2^{(\mathcal{U})}} \bigwedge_{x \in X}\left(G^{\prime}(x) \vee \bigvee_{F \in \mathcal{V}} F(x)\right)
$$

Hence $\forall a \in M(L)$ and for every countable family $\mathcal{U} \subseteq \mathcal{T}_{[a]}$, we have that

$$
a \leq \bigwedge_{x \in X}\left(G^{\prime}(x) \vee \bigvee_{F \in \mathcal{U}} F(x)\right) \Rightarrow a \leq \bigvee_{\mathcal{V} \in 2^{(\mathcal{U})}} \bigwedge_{x \in X}\left(G^{\prime}(x) \vee \bigvee_{F \in \mathcal{V}} F(x)\right)
$$

Thus $\forall b \in \beta(a)$, there exists $\mathcal{V} \in 2^{(\mathcal{U})}$ such that $b \leq \bigwedge_{x \in X}\left(G^{\prime}(x) \vee \bigvee_{F \in \mathcal{V}} F(x)\right)$, i.e., $\forall a \in M(L), \forall b \in \beta(a)$, each countable $Q_{a}$-cover $\mathcal{U}$ of $G$ in $\left(X, \mathcal{T}_{[a]}\right)$ has a finite subfamily $\mathcal{V}$ which is a $Q_{b}$-cover of $G$. Therefore $\forall a \in M(L), G$ is countably a-compact in $\left(X, \mathcal{T}_{[a]}\right)$.

(Sufficiency) Suppose that $\forall a \in M(L), G$ is countably a-compact in $\left(X, \mathcal{T}_{[a]}\right)$. Let $\mathcal{U} \subseteq L^{X}(\mathcal{U}$ is countable family $)$ and $a \leq \bigwedge_{F \in U} \mathcal{T}(F) \wedge$ $\left(\bigwedge_{x \in X}\left(G^{\prime}(x) \vee \bigvee_{F \in \mathcal{U}} F(x)\right)\right)$. Then $\mathcal{U} \subseteq \mathcal{T}_{[a]}$ and $a \leq \bigwedge_{x \in X}\left(G^{\prime}(x) \vee \bigvee_{F \in \mathcal{U}} F(x)\right)$ Thus $\forall b \in \beta(a)$, there exists $\mathcal{V} \in 2^{(\mathcal{U})}$ such that $b \leq \bigwedge_{x \in X}\left(G^{\prime}(x) \vee \bigvee_{F \in \mathcal{V}} F(x)\right)$. Hence $a \leq \bigvee_{\mathcal{V} \in 2^{(\mathcal{U})}} \bigwedge_{x \in X}\left(G^{\prime}(x) \vee \bigvee_{F \in \mathcal{V}} F(x)\right)$. Therefore $G$ is $L$-fuzzy countably compact in $(X, \mathcal{T})$.

Analogous to Shi's proof in [8], we can obtain the following Lemma 2.7.

Lemma 3.9. Let $(X, \mathcal{T})$ be an $L$-topological space, $a \in M(L)$ and $G \in$ $L^{X}$. If $G$ is countably a-compact, then $G \wedge H$ is countably a-compact for each $H \in \mathcal{T}^{\prime}$.

Theorem 3.10. Let $(X, \mathcal{T})$ be an $L$-fuzzy topological space and $G \in L^{X}$. If $G$ is $L$-fuzzy countably compact, then for each $H \in L^{X}$ with $\mathcal{T}^{*}(H)=\top$, $G \wedge H$ is $L$-fuzzy countably compact.

Proof. $\forall a \in M(L)$, since $G$ is $L$-fuzzy countably compact in $(X, \mathcal{T})$, by Theorem 2.6, $G$ is countably $a$-compact in $\left(X, \mathcal{T}_{[a]}\right)$. By $\mathcal{T}^{*}(H)=\top$, we 
know that $H \in \mathcal{T}_{[a]}^{\prime}$. Further by Lemma 2.7, $G \wedge H$ is countably $a$-compact in $\left(X, \mathcal{T}_{[a]}\right)$. Then by Theorem $2.8, G \wedge H$ is $L$-fuzzy countably compact in $(X, \mathcal{T})$.

Analogous to Shi's proof in [8], we can obtain the following Lemma 2.9 .

Lemma 3.11. Let $(X, \mathcal{T})$ be an $L$-topological space, $G, H \in L^{X}$ and $a \in$ $M(L)$. If $G$ and $H$ are countably a-compact, then $G \vee H$ is countably a-compact as well.

Theorem 3.12. Let $(X, \mathcal{T})$ be an $L$-fuzzy topological space and $H, G \in$ $L^{X}$. If $G$ and $H$ are $L$-fuzzy countably compact, then $G \vee H$ is $L$-fuzzy countably compact as well.

Proof. Since both $G$ and $H$ are $L$-fuzzy countably compact in $(X, \mathcal{T})$, by Theorem 2.6, $\forall a \in M(L)$, we know that both $G$ and $H$ are countably a-compact in $\left(X, \mathcal{T}_{[a]}\right)$. By Lemma $2.9, G \vee H$ is countably a-compact in $\left(X, \mathcal{T}_{[a]}\right)$. So $G \vee H$ is $L$-fuzzy countably compact in $(X, \mathcal{T})$.

Analogous to Shi's proof in [8] , we can obtain the following Lemma 2.11 .

Lemma 3.13. Let $(X, \mathcal{T}),(Y, \mathcal{U})$ be two $L$-topological spaces and a $\in$ $M(L)$. If $G$ is countably a-compact in $(X, \mathcal{T})$ and $f:(X, \mathcal{T}) \rightarrow(Y, \mathcal{U})$ is an $L$-continuous mapping, then $f_{L}(G)$ is countably a-compact in $(Y, \mathcal{U})$.

Theorem 3.14. Let $(X, \mathcal{T}),(Y, \mathcal{U})$ be two $L$-fuzzy topological spaces, and $f:(X, \mathcal{T}) \rightarrow(Y, \mathcal{U})$ be an $L$-fuzzy continuous mapping. If $G \in L^{X}$ is $L$-fuzzy countably compact in $(X, \mathcal{T})$, then so is $f_{L}(G)$ in $(Y, \mathcal{U})$.

Proof. Since $G$ is $L$-fuzzy countably compact in $(X, \mathcal{T})$, by Theorem 2.6, $\forall a \in M(L), G$ is countably a-compact in $\left(X, \mathcal{T}_{[a]}\right)$. By Theorem 1.2, $f:\left(X, \mathcal{T}_{[a]}\right) \rightarrow\left(Y, \mathcal{U}_{[a]}\right)$ is an L-continuous mapping. Hence $f_{L}(G)$ is countably a-compact in $\left(Y, \mathcal{U}_{[a]}\right)$. Therefore $f_{L}(G)$ is L-fuzzy countably compact in $(Y, \mathcal{U})$.

\section{The $L$-fuzzy Lindelöf property}

Definition 4.1. Let $(X, \mathcal{T})$ be an $L$-fuzzy topological space. $G \in L^{X}$ is said to have the $L$-fuzzy Lindelöf property if for every family $\mathcal{U} \subseteq L^{X}$, it 
follows that

$$
\bigwedge_{F \in \mathcal{U}} \mathcal{T}(F) \wedge\left(\bigwedge_{x \in X}\left(G^{\prime}(x) \vee \bigvee_{F \in \mathcal{U}} F(x)\right)\right) \leq \bigvee_{\mathcal{V} \in 2^{[\mathcal{U}]}} \bigwedge_{x \in X}\left(G^{\prime}(x) \vee \bigvee_{F \in \mathcal{V}} F(x)\right)
$$

Obviously we have the following theorem.

Theorem 4.2. Let $(X, \mathcal{T})$ be an $L$-fuzzy topological space and $G \in L^{X}$ has the $L$-fuzzy Lindelöf property. Then $G$ is L-fuzzy compact if and only if it is $L$-fuzzy countably compact.

Analogous to $L$-fuzzy countable compactness, we have the following results.

Theorem 4.3. Let $(X, \mathcal{T})$ be an $L$-topological space and $G \in L^{X}$. G has the $L$-fuzzy Lindelöf property in $\left(X, \chi_{\mathcal{T}}\right)$ if and only if $G$ has the Lindelöf property in $(X, \mathcal{T})$.

Theorem 4.4. Let $(X, \mathcal{T})$ be an $L$-fuzzy topological space. $G \in L^{X}$ has the $L$-fuzzy Lindelöf property if and only if for every family $\mathcal{P} \subseteq L^{X}$, it follows that

$$
\bigvee_{F \in \mathcal{P}} \mathcal{T}^{\prime}\left(F^{\prime}\right) \vee\left(\bigvee_{x \in X}\left(G(x) \wedge \bigwedge_{F \in P} F(x)\right)\right) \geq \bigwedge_{\mathcal{H} \in 2^{[P]}} \bigvee_{x \in X}\left(G(x) \wedge \bigwedge_{F \in H} F(x)\right) .
$$

Theorem 4.5. Let $(X, \mathcal{T})$ be an $L$-fuzzy topological space and $G \in L^{X}$. Then the following conditions are equivalent to each other.

(1) G has the L-fuzzy Lindelöf property.

(2) For any $a \in M(L)$, each strong a-remote family $\mathcal{P}$ of $G$ with $\bigwedge_{F \in P} \mathcal{T}^{*}(F) \not{Z}$ $a^{\prime}$ has a countable subfamily $\mathcal{H}$ which is a (strong) a-remote family of $G$.

(3) For any $a \in M(L)$, and any strong a-remote family $\mathcal{P}$ of $G$ with $\bigwedge_{F \in P} \mathcal{T}^{*}(F) \not \leq a^{\prime}$, there exists a countable subfamily $\mathcal{H}$ of $\mathcal{P}$ and $b \in$ $\beta^{*}(a)$ such that $\mathcal{H}$ is a (strong) b-remote family of $G$.

(4) For any $a \in P(L)$, each strong a-shading $U$ of $G$ with $\bigwedge_{F \in \mathcal{U}} \mathcal{T}(F) \not \leq a$ has a countable subfamily $\mathcal{V}$ which is a (strong) a-shading of $G$. 
(5) For any $a \in P(L)$ and any strong a-shading $\mathcal{U}$ of $G$ with $\bigwedge_{F \in U} \mathcal{T}(F) \not{Z}$ $a$, there exists a countable subfamily $\mathcal{V}$ of $\mathcal{U}$ and $b \in \alpha^{*}(a)$ such that $\mathcal{V}$ is a (strong) b-shading of $G$.

(6) For any $a \in M(L)$ and any $b \in \beta^{*}(a)$, each $Q_{a}$-cover $\mathcal{U}$ of $G$ with $\mathcal{T}(F) \geq a(\forall F \in \mathcal{U})$ has a countable subfamily $\mathcal{V}$ which is a $Q_{b}$-cover of $G$.

(7) For any $a \in M(L)$ and any $b \in \beta^{*}(a)$, each $Q_{a}$-cover $\mathcal{U}$ of $G$ with $\mathcal{T}(F) \geq a(\forall F \in \mathcal{U})$ has a countable subfamily $\mathcal{V}$ which is a (strong) $\beta_{b}$-cover of $G$.

Theorem 4.6. Let $(X, \mathcal{T})$ be an $L$-fuzzy topological space and $G \in L^{X}$. If $\beta(c \wedge d)=\beta(c) \cap \beta(d)(\forall c, d \in L)$, then the following conditions are equivalent to each other.

(1) $G$ has the L-fuzzy Lindelöf property.

(2) For any $a \in M(L)$, each strong $\beta_{a}$-cover $\mathcal{U}$ of $G$ with $a \in \beta\left(\bigwedge_{F \in \mathcal{U}} \mathcal{T}(F)\right)$ has a countable subfamily $\mathcal{V}$ which is a (strong) $\beta_{a}$-cover of $G$.

(3) For any $a \in M(L)$ and any strong $\beta_{a}$-cover $\mathcal{U}$ of $G$ with

$a \in \beta\left(\bigwedge_{F \in \mathcal{U}} \mathcal{T}(F)\right)$, there exists a countable subfamily $\mathcal{V}$ of $\mathcal{U}$ and $b \in M(L)$ with $a \in \beta^{*}(b)$ such that $\mathcal{V}$ is a (strong) $\beta_{b}$-cover of $G$.

Definition 4.7. Let $(X, \mathcal{T})$ be an $L$-topological space, $a \in M(L)$ and $G \in$ $L^{X} . G$ has the a-Lindelöf property if and only if $\forall b \in \beta(a)$, each $Q_{a}$-open cover $\mathcal{U}$ of $G$ has a countable subfamily $\mathcal{V}$ which is a $Q_{b}$-open cover of $G$.

Theorem 4.8. Let $(X, \mathcal{T})$ be an $L$-topological space. Then $G \in L^{X}$ has the Lindelöf property if and only if $\forall a \in M(L), G$ has the a-Lindelöf property.

Theorem 4.9. Let $(X, \mathcal{T})$ be an $L$-fuzzy topological space and $G \in L^{X}$. Then $G$ has the L-fuzzy Lindelöf property in $(X, \mathcal{T})$ if and only if $\forall a \in$ $M(L), G$ has the a-Lindelöf property in $\left(X, \mathcal{T}_{[a]}\right)$.

Lemma 4.10. Let $(X, \mathcal{T})$ be an $L$-topological space, $a \in M(L)$ and $G \in$ $L^{X}$. If $G$ has the $a$ - Lindelöf property, then $G \wedge H$ has the a-Lindelöf property for each $H \in \mathcal{T}^{\prime}$. 
Theorem 4.11. Let $(X, \mathcal{T})$ be an $L$-fuzzy topological space and $G \in L^{X}$. If $G$ has the $L$-fuzzy Lindelöf property, then for each $H \in L^{X}$ with $\mathcal{T}^{*}(H)=$ Т, $G \wedge H$ has the L-fuzzy Lindelöf property.

Lemma 4.12. Let $(X, \mathcal{T})$ be an $L$-topological space, $G, H \in L^{X}$ and $a \in$ $M(L)$. If $G$ and $H$ have the a-Lindelöf property, then $G \vee H$ has the $a$ Lindelöf property as well.

Theorem 4.13. Let $(X, \mathcal{T})$ be an $L$-fuzzy topological space and $H, G \in$ $L^{X}$. If $G$ and $H$ have the $L$-fuzzy Lindelöf property, then $G \vee H$ has the $L$-fuzzy Lindelöf property as well.

Lemma 4.14. Let $(X, \mathcal{T}),(Y, \mathcal{U})$ be two L-topological spaces and a $\in$ $M(L)$. If $G$ has the a-Lindelöf property in $(X, \mathcal{T})$ and $f:(X, \mathcal{T}) \rightarrow(Y, \mathcal{U})$ is an $L$-continuous mapping, then $f_{L}(G)$ has the a-Lindelöf property in $(Y, \mathcal{U})$.

Theorem 4.15. Let $(X, \mathcal{T}),(Y, \mathcal{U})$ be two L-fuzzy topological spaces, and $f:(X, \mathcal{T}) \rightarrow(Y, \mathcal{U})$ be an $L$-fuzzy continuous mapping. If $G \in L^{X}$ has the $L$-fuzzy Lindelöf property in $(X, \mathcal{T})$, then $f_{L}(G)$ has the L-fuzzy Lindelöf property in $(Y, \mathcal{U})$.

\section{References}

[1] P. Dwinger, Characterizations of the complete homomorphic images of a completely distributive complete lattice I, Indagationes Mathematicae (Proceedings), 85, pp. 403-414, (1982).

[2] U. Höhle, A.P. S̆ostak, Axiomatic foundations of fixed-basis fuzzy topology, Chapter 3 in: U. Höhle, S.E. Rodabaugh (Eds), Mathematics of Fuzzy Sets: Logic, Topology, and Measure Theory, Kluwer Academic Publishers (Boston/Dordrecht/London), 1999.

[3] T. Kubiak, On fuzzy topologies, Ph. D. Thesis, Adam Mickiewicz, Poznan, Poland, (1985).

[4] Y. M. Liu, M. K. Luo, Fuzzy topology, World Scientific, Singapore, (1997). 
[5] R. Lowen, Fuzzy topological spaces and fuzzy compactness. J. Math. Anal. Appl., 56, pp- 621-633, (1976).

[6] S. E. Rodabaugh, Categorical foundations of variable-basis fuzzy topology, Chapter 4 in [2].

[7] F.-G. Shi, A note on fuzzy compactness in $L$-topological spaces, Fuzzy Sets and Systems, 199, pp. 547-548, (2001).

[8] F.-G. Shi, Countable compactness and the Lindelöf property of $L$-fuzzy sets, Iranian Journal of Fuzzy Systems, 1, pp. 79-88, (2004).

[9] F. -G. Shi, A new definition of fuzzy compactness, Fuzzy Sets and Systems, 158, pp. 1486-1495, (2007).

[10] F. -G. Shi, R. -X. Li, Compactness in $L$-fuzzy topological spaces, submitted.

[11] A. P. Sostak, On a fuzzy toplogical structure, Suppl. Rend. Circ. Mat. Palermo Ser., 1, pp. 89-103, (1985).

[12] G. L. Wang, A new fuzzy compactness definied by fuzzy nets, J. Math. Anal. Appl., 94, pp. 1-23, (1983).

[13] G. J. Wang, Theory of L-fuzzy topological space, Shaanxi Normal University Publishers, Xian, (1988). (in Chinese).

[14] J. Zhang, F.-G. Shi and C.-Y. Zheng, On $L$-fuzzy topological spaces, Fuzzy Sets and Systems, 149, pp. 473-484, (2005,).

\section{Run - Xiang Li}

Department of Mathematics, School of Science, Beijing Institute of Technology, Beijing 100081, P. R. China e-mail: lirunxiang84@sina.com

and 


\section{Fu - Gui Shi}

Department of Mathematics,

School of Science,

Beijing Institute of Technology,

Beijing 100081,

P. R. China

e-mail : fuguishi@bit.edu.cn 